\title{
Robotic pancreatic surgery: a slowly developing field
}

\author{
Patrick N. Salibi, John B. Martinie \\ Division of Hepatobiliary and Pancreatic Surgery, Department of Surgery, Atrium Health Carolinas Medical Center, Charlotte, NC, USA \\ Correspondence to: John B. Martinie. Division of Hepatobiliary and Pancreatic Surgery, Atrium Health - Carolinas Medical Center, 1025 Morehead \\ Medical Dr, Suite 600, Charlotte, NC 28204, USA. Email: John.martinie@atriumhealth.org. \\ Comment on: Liu R, Wakabayashi G, Palanivelu C, et al. International consensus statement on robotic pancreatic surgery. Hepatobiliary Surg Nutr \\ 2019;8:345-60.
}

Submitted Jan 07, 2020. Accepted for publication Feb 06, 2020.

doi: 10.21037/hbsn.2020.02.02

View this article at: http://dx.doi.org/10.21037/hbsn.2020.02.02

Robotic surgery has undergone rapid development over the last several decades and minimally invasive surgery has become the standard of care for many surgical specialties. Its adoption within the world of hepatobiliary and pancreas surgery has been tempered by the complexity of the procedures. Early reports showcased the feasibility of distal pancreatectomy and pancreaticoduodenectomy in a minimally invasive fashion and where followed by case series demonstrating its safety and efficacy $(1,2)$. This has since been confirmed with large matched cohorts confirming at a minimum oncologic equivalence between minimally invasive pancreatic resections as compared to open procedures (3). Given the complexity of pancreatic surgery and the inherent limitations of laparoscopic surgery the robotic surgical platform has seen an exponential rise in attention with its role in minimally invasive pancreatic resections. Given this interest there have been two consensus guidelines published on minimally invasive pancreatic surgery demonstrating its growth within the field $(4,5)$.

The most recent publication reviewing this topic by Liu et al. composed a consensus document on robotic pancreatic surgery (4). The aim of this consensus statement was to review the safety, feasibility, efficacy and costeffectiveness of this approach in order to provide guidance and make recommendations for its future development. The committee, using the process established by the World Health Organization Handbook for Guideline Development, determined the topics of interest, prepared evidence-based documents and generated final recommendations. This was completed using the well-established and broadly used GRADE (Grading of Recommendations Assessment, Development and
Evaluation) approach to assess the quality of evidencebased literature supporting each topic along with the Delphi process to obtain expert consensus on statements. With this methodology the committee proposed 19 topics that were analyzed producing 16 recommendations assessed by GRADE and 3 recommendations that lacked sufficient evidence-based literature to make formal GRADE recommendations but provided literature review and expert panel opinion on.

The first 6 recommendations focused on robotic distal pancreatectomy (RDP) as it compares to both laparoscopic (LDP) and open distal pancreatectomy (ODP). The committee provided strong recommendation for the use of the robotic platform for this procedure regarding safety, feasibility and efficacy with a mix of moderate to low levels of evidence to support this. LDP has become standard practice and thus providing evidence-based support for RDP as at least equivalent to LDP is essential to its ongoing practice. The committee found since the first case presented in 2001 there has been a gradual accrual of case reports and more recent case series, meta-analyses and RCTs showing comparable outcomes and peri-operative measures of feasibility between RDP, LDP and ODP (6-8). They focused on the evidence that points towards similar lymph node harvest and surgical margin status as well as overall survival noted in the RDP vs. LDP and OPD. Evidence was also presented with commendation for RDP given its comparable postoperative outcomes and morbidity with decreased length of stay and intraoperative blood loss. Our own experience and data comparing robotic versus the laparoscopic approach for distal pancreatectomy reiterated the comparable outcomes in morbidity, mortality and 
oncologic outcomes between the two minimally invasive approaches. Again the consensus committee recommended strongly for the use of RDP over LDP with low level of evidence when splenic preservation is planned as RDP improves the rate of splenic vessel preservation (Kimura method) as compared to LDP which had similar rates of splenic preservation in meta-analysis but was less likely to preserve the splenic vessels (Warshaw method) $(8,9)$. As such when splenic parenchymal and vessel preservation is desired RDP may provide the tools to facilitate the delicate dissection necessary to achieve this more consistently. The portion of the article focusing on RDP provided the strongest level of evidence for the statements made throughout the piece. This likely reflects the increased case experience of surgeons with the robotic platform performing RDP as compared to other procedures resulting in an increased volume and quality of data for this specific surgery that exceeds the remaining topics addressed in this paper. In spite of this, the highest level of evidence achieved in this article was moderate even for RDP at best.

Several recommendations through out the paper focused on both RDP as well as robotic pancreaticoduodenectomy (RPD) and the anticipated learning curves to obtain the skills necessary to be deemed proficient and have improved outcomes. The number 10-20 cases used for RDP was given based on the limited number amount of literature available. The same appeared true for RPD in which several articles seemed to point towards 40 cases as a relative turning point in the observed operative time and postoperative outcomes. The difficulty in determining a set case volume for learning curves by the committee is due to the paucity of evidence to support these thresholds and most research published in this regard is based on single or multi-surgeon case series. The articles cited did use cumulative sum analysis to objectively assess their progress but was retrospective in nature. It is apparent that prospective structured research is needed in this area in order to better established expectations and benchmarks for those desiring to begin to utilize the robotic platform in their practice.

Also included was recommendations on several procedures that could be performed on the robot but were not currently recommended by the committee based on the lack of evidence indicating improved outcomes or need. The first of the procedures discussed was radical antegrade modular pancreatosplenectomy (RAMPS) and based on a limited number of published cases it appears feasible and safe to perform robotically but given the lack of strong evidence showing long term oncologic benefit we would agree with the authors in noting that surgeon preference and ongoing research will determine its utility with the robotic platform in the future (10). The authors also address RPD in the setting of vascular resection and reconstruction stating with weak recommendation and very low level of evidence that portal vein and superior mesenteric vein resections and reconstruction are difficult procedures and should not be performed in the preliminary stages of training on the robotic platform. They report on 4 case series demonstrating the feasibility of the procedure but note the emphasis on increased blood loss and the limited number of cases which they rightly state indicates complexity and difficulty of the case. This is a procedure that is fraught with the risk of uncontrolled hemorrhage and as such is not at a point were it should be performed regularly in a robotic fashion particularly by those in the beginning or intermediate phases of their robotic experience. The committee also evaluated central pancreatectomies and based on the limited amount of work published in this area found no compelling evidence to indicated improved outcomes or preoperative recovery when performing this robotically as compared to open. It is likely that given the procedure is not nearly as common as the distal pancreatectomy or pancreaticoduodenectomy the benefits of minimally invasive surgery likely still translate to a shorter hospital length of stay and improved recovery even though the data has yet to demonstrate this given its spareness. Lastly the committee touched on enucleation procedures and the utility of the robot in this procedure. Again while there is not a large volume of evidence to show superiority on the robotic platform it has been demonstrated to be safe and feasibly to perform while adherence to prior established guidelines about size of lesion and distance from the main pancreatic duct appear to hold true. Enucleation appears to be a ideal application of the robotic platform due to the greatly improved visualization with video quality as well as the improved dexterity in performing fine dissection in a small operative field.

Any discussion of robotic surgery would be incomplete without an attempt to evaluate the cost effectiveness of the approach. As with many areas of robotic surgery attempts to quantify this have been hampered by differences in assessments of cost (operating room costs vs hospitalization costs) as well as regional variations in the cost of delivery of healthcare (11). The literature appears conflicting and inconclusive in regards to this. To complicate this further is the fact that as the robotic platform becomes more common place the monopoly currently held by a single 
company on robotic intra-peritoneal surgery will soon be lost as several other companies are moving quickly towards viable alternatives. This competition will ultimately drive down pricing making robotic procedures more cost competitive.

The paper provides a thorough review and salient expert interpretation of the current literature regarding robotic pancreas surgery and its rapid development over the last few decades. The overall sense that the paper provides is that there is a paucity of quality data on this topic. This should be a call to the pancreatic surgery community, especially those who believe in the technology and principles of minimally invasive and robotic surgery, to view these recommendations as areas to focus future high quality research in to validate these points.

\section{Acknowledgments}

Funding: None.

\section{Footnote}

Provenance and Peer Review: This article was commissioned by the editorial office, Hepatobiliary Surgery and Nutrition. The article did not undergo external peer review.

Conflicts of Interest: The authors have no conflicts of interest to declare.

Ethical Statement: The authors are accountable for all aspects of the work in ensuring that questions related to the accuracy or integrity of any part of the work are appropriately investigated and resolved.

Open Access Statement: This is an Open Access article distributed in accordance with the Creative Commons Attribution-NonCommercial-NoDerivs 4.0 International License (CC BY-NC-ND 4.0), which permits the noncommercial replication and distribution of the article with the strict proviso that no changes or edits are made and the original work is properly cited (including links to both the formal publication through the relevant DOI and the license).
See: https://creativecommons.org/licenses/by-nc-nd/4.0/.

\section{References}

1. Gagner M, Pomp A. Laparoscopic pylorus-preserving pancreatoduodenectomy. Surg Endosc 1994;8:408-10.

2. Kooby DA, Gillespie T, Bentrem D, et al. Left-sided Pancreatectomy: A Multicenter Comparison of Laparoscopic and Open Approaches. Ann Surg 2008;248:438-46.

3. Kauffmann EF, Napoli N, Menonna F, et al. A propensity score-matched analysis of robotic versus open pancreatoduodenectomy for pancreatic cancer based on margin status. Surg Endosc 2019;33:234-42.

4. Liu R, Wakabayashi G, Palanivelu C, et al. International consensus statement on robotic pancreatic surgery. Hepatobiliary Surg Nutr 2019;8:345-60.

5. Asbun HJ, Moekotte AL, Vissers FL, et al. The Miami International Evidence-based Guidelines on Minimally Invasive Pancreas Resection. Ann Surg 2020;271:1-14.

6. Melvin WS, Needleman BJ, Krause KR, et al. Computerenhanced robotic telesurgery. Initial experience in foregut surgery. Surg Endosc 2002;16:1790-2.

7. Zhao W, Liu C, Li S, et al. Safety and efficacy for robotassisted versus open pancreaticoduodenectomy and distal pancreatectomy: A systematic review and meta-analysis. Surg Oncol 2018;27:468-78.

8. Lyman WB, Passeri M, Sastry A, et al. Robotic-assisted versus laparoscopic left pancreatectomy at a high-volume, minimally invasive center. Surg Endosc 2019;33:2991-3000.

9. Xu SB, Jia CK, Wang JR, et al. Do patients benefit more from robot assisted approach than conventional laparoscopic distal pancreatectomy? A meta-analysis of perioperative and economic outcomes. J Formos Med Assoc 2019;118:268-78.

10. Zhou Q, Fengwei G, Gong J, et al. Assessement of postoperative long-term survival quality and complications associated with radical antegrade modular pancreatosplenectomy and distal pancreatectomy: a metaanalysis and systematic review. BMC Surg 2019;19:12.

11. Patti JC, Ore AS, Barrows C, et al. Value-based assessment of robotic pancreas and liver surgery. Hepatobiliary Surg Nutr 2017;6:246-57.
Cite this article as: Salibi PN, Martinie JB. Robotic pancreatic surgery: a slowly developing field. HepatoBiliary Surg Nutr 2020;9(5):673-675. doi: 10.21037/hbsn.2020.02.02 